\title{
Anaesthetic Management of Emergency Tracheostomy in a Three Years Old Child with Recurrent Laryngeal Papillomatosis: A Case Report
}

\author{
Dr. Prakash Deb ${ }^{1}$, Dr. Md. Yunus ${ }^{2}$, Dr. Narendra Kumar Sharma ${ }^{3}$ \\ ${ }^{1}$ Senior Resident, Department of Anaesthesia, Critical Care and Pain, NEIGRIHMS, Shillong, India \\ ${ }^{2}$ Additional Professor, Department of Anaesthesia, Critical Care and Pain, NEIGRIHMS, Shillong, India \\ ${ }^{3}$ Junior Resident, Department of Anaesthesia, Critical Care and Pain, NEIGRIHMS, Shillong, India \\ Corresponding author: Dr. Prakash Deb, Quarter B8F, NEIGRIHMS Campus, NEIGRIHMS, Shillong, India; \\ prox.deb@gmail.com
}

Received 24 May 2020;

Accepted 22 June 2020;

Published 04 July 2020

\begin{abstract}
Juvenile onset recurrent laryngeal papillomatosis is a condition which requires multiple surgical excisions to maintain airway patency. However, if neglected may lead to airway compromise requiring emergency tracheostomy and anaesthetic management in such paediatric case is challenging. Awake tracheostomy is difficult and risky in children who are usually restless and uncooperative. Also induction of anaesthesia by intravenous or inhalational route pose a threat to the loss of airway muscle tone \& patency. We present here a three years old child with recurrent laryngeal papillomatosis who landed up with emergency tracheostomy for severe stridor where the passage of even the smallest size endotracheal tube would not be possible however emergency tracheostomy could be safely done by inhalational induction maintaining spontaneous ventilation. Such paediatric case where even endotracheal tube could not be passed has been rarely reported. Also literature on anaesthetic management of paediatric emergency tracheostomy is sparse.
\end{abstract}

Keywords: recurrent laryngeal papillomatosis, paediatric, emergency tracheostomy, anaesthesia

\section{Background}

Juvenile onset recurrent laryngeal papillomatosis is a condition which requires multiple surgical excisions to maintain airway patency ${ }^{[1]}$. However if neglected may lead to airway compromise requiring emergency tracheostomy and anaesthetic management in such paediatric case is challenging. Awake tracheostomy in children is difficult \& risky. Also induction of anaesthesia with intravenous or inhalational route pose a threat to the loss of airway muscle tone \& patency. We present here a three years old child with recurrent laryngeal papillomatosis who landed up with emergency tracheostomy for severe stridor which could be safely done by inhalational induction maintaining spontaneous ventilation.

\section{Case History}

A three years old boy presented to our Emergency Department with severe stridor. The child was already a diagnosed case of recurrent laryngeal papillomatosis operated thrice in another hospital in the preceding seven months, the last operation being done three months back. However the patient did not go for subsequent follow up.

Two months following the last surgery the child started developing stridor gradually which worsened on the day he presented to emergency department. The stridor was biphasic and associated with involvement of all the accessory respiratory muscles. There was intercostal, subcostal \& sub-sternal retraction, wrinkling of forehead, elevation of both the upper eyelids during inspiratory efforts. On general examination the child weighed $12 \mathrm{~kg}$, there was no sign of cyanosis, PR: 106/min, BP: 90/48mmHg, RR: 20/min. The child had chest deformity (pectus excavatum). There was no scar of tracheostomy.

There was a report of fibre optic laryngoscopy done prior to the first surgery which showed growth covering laryngeal inlet with $1 \mathrm{~mm}$ of aperture left. An X-ray neck lateral view done two days back showed a clear soft tissue mass in the laryngeal area extending to subglottic region, with a thin column of air (even $<1$ $\mathrm{mm})$. Figure 1. 


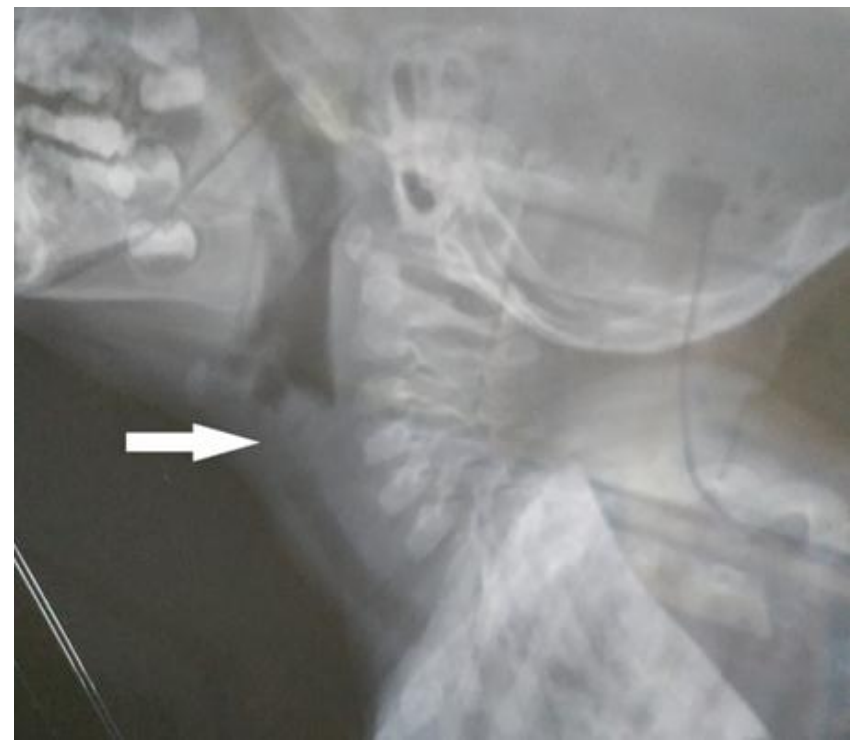

Figure 1: Lateral $\mathrm{X}$-ray neck showing laryngeal growth (marked by arrow)

The child was irritable and restless. Fibre optic laryngoscopy or indirect laryngoscopy was neither possible nor helpful at that stage. Patient was immediately shifted to OT for proper airway management. 22G intravenous cannula secured. Considering the clinical status, presence of severe stridor, near complete occlusion of larynx \& trachea in X-ray neck, decision for upfront tracheostomy was taken after discussion between senior otorhinolaryngologist and anaesthesiologist. Appropriate sized tracheostomy tubes, needle crico-thyrotomy set, small size bougie, guidewire, jet ventilation, paediatric fibreoptic bronchoscope, rigid bronchoscope, endotracheal tubes of different sizes starting from smallest possible ones were on the emergency airway trolley in addition to all the conventional equipments. Drugs \& equipments necessary for proper resuscitation were kept ready according to age $\&$ weight.

As the patient was restless, agitated \& uncooperative fast induction with inhalational agent was done using $100 \% \mathrm{O}_{2}$ with $8 \%$ Sevoflurane via face mask maintaining spontaneous ventilation. Immediately following induction the concentration of sevoflurane was reduced and titrated to maintain anaesthetic depth and spontaneous ventilation. Slight head up position $\left(30^{\circ}\right)$ was maintained. Local infiltration of the incision area was done with Inj. Lignocaine $2 \%(1.5 \mathrm{ml})$. Surgical tracheostomy was done using $4.5 \mathrm{~mm}$ ID cuffed tracheostomy tube between $2^{\text {nd }} \& 3^{\text {rd }}$ tracheal ring via vertical incision and confirmed using capnography. The patient was given Inj. Vecuronium $1 \mathrm{mg}$ intravenous and anaesthesia was maintained using $\mathrm{O}_{2}+$ Sevoflurane \& controlled mechanical ventilation. An attempt was made to resect the growth per orally following fibre optic laryngoscopy. However as anatomical structures could not be identified due to extensive growth involving the supra-glottic part (figure 2) surgical procedure was abandoned and planned for future surgical resection after thorough evaluation by CT scan. Patient was successfully reversed \& sent to ICU in room air on spontaneous ventilation via tracheostomy.

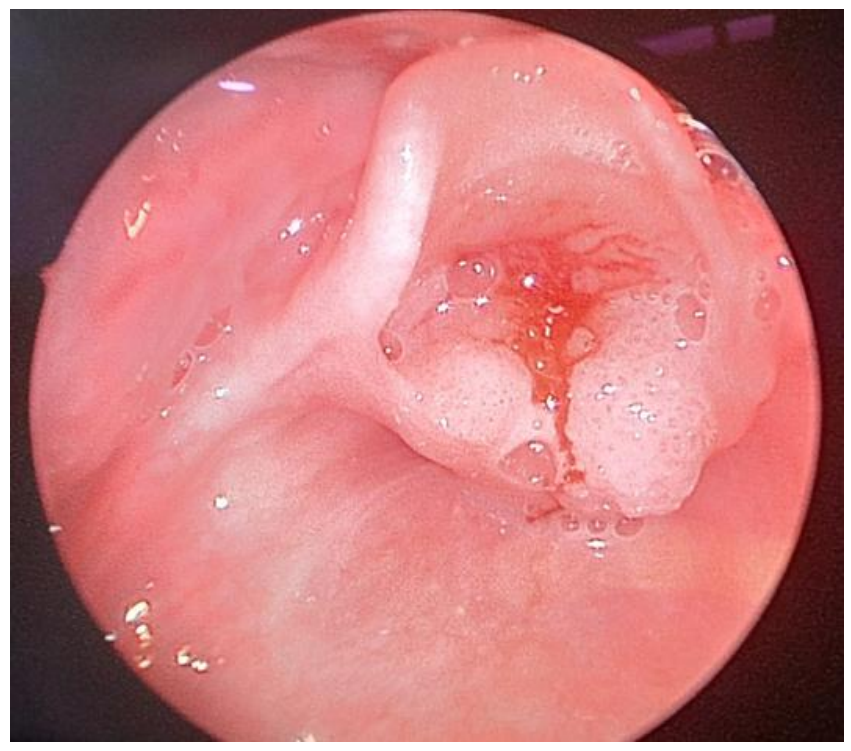

Figure 2: Fibreoptic laryngoscopic view showing near complete obstruction of laryngeal inlet.

\section{Discussion}

Recurrent laryngeal papillomatosis, caused by Human Papilloma Virus 6 \& 11, has an aggressive nature and usually requires multiple surgical excisions to maintain airway patency and voice quality ${ }^{[1]}$. Occasionally adjuvants like interferon, celecoxib, cidofovir, bevacizumab are required in some cases ${ }^{[2]}$. In juvenile onset disease however the need for multiple surgery significantly reduces after 15 years of age ${ }^{[3]}$. Tracheostomy is avoided unless there is life threatening airway occlusion as tracheostomy may be a route of distal spread of the disease to lower respiratory tract \& site for rapid colonisation.

In this case the three years old child was operated multiple times but didn't turn up for follow up until stridor became severe enough to require emergency tracheostomy. Considering the severity of stridor and airway narrowing evident on $\mathrm{x}$-ray neck, passage of smallest endotracheal tube would not be possible and an attempt of securing airway via endotracheal tube seemed risky as minimal instrumentation of upper airway could cause oedema, injury, bleeding, aspiration \& laryngospasm totally occluding the already compromised airway. The depth of anaesthesia required to make this trial of endotracheal tube placement might cause loss of upper airway tone which could be catastrophic. Awake tracheostomy although a safer approach was not feasible in this case as the child was restless and uncooperative. There is insufficient literature on awake tracheostomy in paediatric patient. One case reported by Ian Yuan et al. described emergency awake tracheostomy using minimal sedation but in that case the 7 years old child was calm and cooperative even before starting sedation ${ }^{[4]}$.

Induction with inhalational agent in our patient with such severe stridor could be disastrous due to worsening airway obstruction resulting from loss of airway tone as anaesthesia deepens. Proper positioning with jaw thrust \& positive airway pressure upto $10 \mathrm{cmH} 2 \mathrm{o}$ could be beneficial. Considering the magnitude of narrowed airway measures for apnoeic oxygenation like needle cricothyrotomy was kept ready. However, procedure was successfully done using inhalational induction while maintaining spontaneous ventilation \& the need of cricothyrotomy didn't arise. In such critical scenario a multidisciplinary approach involving anaesthesiologist, paediatrician, otorhinolaryngologist is 
required. Proper communication amongst them \& OT staff with appropriate backup measures is of utmost importance.

Anaesthetic management of emergency tracheostomy in paediatric patient is extremely challenging and literature on the same is sparse. Further research in such cases may help come up with a safer anaesthetic management protocol.

\section{References}

[1] Reeves WC, Ruparelia SS, Swanson KI, Derkay CS, Marcus A, Unger ER. National registry for juvenile-onset recurrent respiratory papillomatosis. Arch Otolaryngol Head Neck Surg 2003;129(9):976-982.
[2] Schraff S, Derkay CS, Burke B, Lawson L. American Society of Pediatric Otolaryngology members' experience with recurrent respiratory papillomatosis and the use of adjuvant therapy. Arch Otolaryngol Head Neck Surg 2004;130(9):1039-1042.

[3] Silverberg MJ, Thorsen P, Lindeberg H, Ahdieh-Grant L, Shah KV. Clinical course of recurrent respiratory papillomatosis in danish children. Arch Otolaryngol Head Neck Surg 2004;130(6):711-716.

[4] Yuan I, Bruins B, Kiell EP, Javia LR. Anesthetic management for pediatric Awake Tracheostomy. 2016 Dec;1;7(11):236-238. 